\title{
STOCHASTIC CHOICE HEURISTICS *
}

\author{
K. Michael ASCHENBRENNER, Dietrich ALBERT and \\ Franz SCHMALHOFER
}

University of Heidelberg, West-Germany

\begin{abstract}
A class of stochastic models is presented which assume that binary choice among multidimensional alternatives is accomplished by sequential accumulation of dimensional value differences until enough evidence is accumulated. The models purpose not only to predict individual choices or choice probabilities from dimensional evaluations of the alternatives but also to map the cognitive processes taking place. The latter conjecture is tested by an analysis of choice latencies. Within the model class competing assumptions are made about the source of fluctuations in choice behavior (fluctuations in values versus fluctuations in the selection of dimensions) and about the dimensional comparison process (cardinal versus ordinal). The models are tested with individual choice probabilities and latencies from eight subjects making many choices between alternatives in four topic fields. The models do better when alternatives are described on a number of dimensions than for alternatives presented only by name. Further, the results suggest that fluctuations in the selection of dimensions must be seriously considered as an alternative to fluctuations in values for explaining choice variability.
\end{abstract}

This study has two objectives: First, to predict individual binary choices among multidimensional alternatives from evaluations of the alternatives' features, that is, their levels on the dimensions, and the importance of the dimensions, and second, to model the cognitive processes leading to these choices.

Choices in applied settings (e.g., in the attitude and behavior context, Fishbein and Ajzen 1975) are often predicted by the weighted additive rule. Recent research on cognitive strategies has revealed, however, that

* This research was supported by Deutsche Forschungsgemeinschaft grant Al 205/1 (SPP: Einstellung und Verhalten). We wish to thank Iris Schaefer, Walter Saffrich, Doris Glavanov, and Joachim Canisius for their help in conducting this research, and Thomas Schneyer for programming the Apple II for running the experiments. The valuable criticisms of Hans Kordy are much appreciated.

Authors' address: Psychologisches Institut, Universitaet Heidelberg, Hauptstrasse 47-51, D-6900 Heidelberg, West-Germany. 
the additive rule usually does not represent the cognitive choice processes. Rather, persons presumably use simple heuristic rules (e.g., Svenson 1979). But these heuristics often do not specify which information will be selected and processed, how long this process will continue, which alternative will be chosen (except for specially designed alternatives, e.g., Huber 1983), and why and when repeated choices from the same alternatives will differ.

In order to answer some of these questions we developed stochastic choice models which assume that heuristic rules are applied to probabilistically selected information. In order to examine whether these models would predict choices and reflect the cognitive processes involved, choice frequencies and choice latencies were analyzed.

\section{General assumptions}

We propose that a binary choice is a sequential comparison process in which alternatives are compared on a number of dimensions. We do not assume, however, that all available or relevant dimensions are used for a choice nor that any fixed number of dimensions is used for choosing among alternatives of a given class. Rather, it is proposed that the comparison process is stopped and an alternative is chosen when a person has accumulated enough evidence to be convinced that one alternative is better than the other.

More specifically, it is assumed that (1) choices are based on sequential dimensional comparisons. The sequence of selecting dimensions for comparison is determined by the importance of the dimensions. (2) For a given dimension the alternatives are compared with respect to the attractiveness of their features. (3) The results of these comparisons are accumulated over dimensions by a counter. (4) This process stops when the counter reaches or exceeds one of two critical values $(k$ or $-k)$ determining which alternative is chosen.

For applying these assumptions to the prediction of choices it is further assumed that (5) the importance of dimensions can be assessed separately. (6) The results of the comparisons on individual dimensions can be estimated from separately assessed evaluations of the alternatives' features. Finally, there is the question of what is enough evidence, that is, the size of the critical values. So far, we have estimated this parameter from the subjects' choice data. It is the only model parameter estimated from the choice data. 


\section{An illustration}

As an illustration consider two arbitrary 11-dimensional alternatives, $x$ and $y$. Assume that the attractiveness values of their features are given by $x:(2,5,2,3,6,2,1,4,7,2,1)$ and $y:(1,7,2,4,3,5,7,6,1,2,4)$. These values are listed in the order from most to least important dimension. Also, assume that positive differences are counted in favor of $x$ and negative ones in favor of $y$ and that the critical values are +3 and -3 . The counter starts at 0 . For the first dimension a difference of 1 in favor of $x$ is found, and so +1 is counted. Since the absolute amount is lower than 3 the second dimension will be processed. Here $y$ is found to be superior by 2 . Thus -2 is added, setting the counter to -1 . For the third dimension, no difference is found, thus the counter stays at -1 . Processing the following dimensions yields differences of $-1,+3,-3,-6$ setting the counter successively to values of $-2,+1$, $-2,-8$. Thus, after the seventh dimension the critical value of -3 is exceeded which stops the process by choosing $y$ since the counter is negative. Therefore, dimensions 8 to 11 will not be considered.

This model not only predicts which alternative will be chosen but also how many dimensions will be processed for making a choice. If the alternative $y$ from the example above had been evaluated 6 rather than 4 on the fourth dimension, the same choice, $y$, would have already resulted after processing the fourth rather than the seventh dimension. We assume that the number of dimensions processed in any choice determines the choice latency.

\section{Specific assumptions of the models}

Within the framework defined above, we made some competing assumptions about the nature of the comparison process and about the possible fluctuations in the choice process.

\section{Comparison modes}

In the illustration given above, cardinal value differences between the two alternatives are accumulated over dimensions; this is called cardinal comparison. There is evidence, however, that persons often determine only which alternative, if any, is better on a given dimension and base 
their choices on these ordinal comparisons (Svenson 1979). Therefore, ordinal models were considered in which the comparison results may only obtain values of $+1,-1$, or 0 , depending on whether $x, y$, or neither alternative is more attractive on a dimension. In the illustration above, ordinal comparison also results in the choice of $y$, but after processing eight rather than seven dimensions. In general, the two comparison modes may yield different choices for the same pairs of alternatives.

\section{Fluctuations in the choice process}

Given a constant set of alternatives, dimensions, evaluations, importance weights, and a critical value, the models developed so far will predict the choice of a fixed alternative for any pair after a fixed number of comparisons. It has often been observed, however, that, given the same alternatives repeatedly, persons do not always make the same choices (e.g., Petrusic and Jamieson 1978). The model has three components that may be subject to fluctuations: (1) The values of a person may be due to some fluctuation, an assumption dating back to Thurstone (1927). (2) The sequence of selecting dimensions may be fluctuating. (3) The critical values may fluctuate. Because no plausible hypotheses were found about fluctuations of the criteria we assumed them to be constant. In the present study it was attempted to compare the competing assumptions about the influence of the fluctuation of values and about the fluctuation of the sequence by which dimensions are processed upon individual choices. For both assumptions a cardinal or an ordinal comparison mode may be assumed, so that four specific models are obtained.

The fluctuation of values was accounted for by the actually observed variation of the evaluations. The resulting models will be called valuefluctuation (VFL) models.

Alternatively, the fluctuation in the selection of dimensions was accounted for in stochastic dimension selection (SDS) models by assuming that the selection of dimensions is an inherently stochastic process which depends on the importance of the dimensions. Although Luce's choice axiom (Luce 1959) is not suited for describing choices between multidimensional alternatives (Restle 1961; Tversky 1972), the selection of dimensions may be well described by this axiom, because importance is considered a single continuum. Let $w_{j}$ be the importance weight of 
dimension $j$, and $J$ be the set of dimensions that have not yet been processed. Then the probability $p_{i}$ that a dimension $i$ will be selected as the next dimension is

$p_{i}=e^{w_{i}} / \sum_{j \in J} e^{w_{j}}$ for $i \in J$ and 0 for $i \notin J .[1]$

\section{The experiment}

The competing assumptions about the source of fluctuations in choice behavior were examined for both comparison modes in an experiment by collecting choice frequencies and latencies elicited from individuals in repeated paired comparisons between realistic alternatives.

Since in realistic settings alternatives are often presented by multidimensional descriptions or by their names, both modes of presentation were employed. Tentatively, we assumed the same cognitive processes for described and named alternatives with dimensions being retrieved from the $S$ 's memory rather than from the display in the latter case.

Also, in many occasions only the decision maker knows the alternatives which are relevant in a given choice situation. If the alternatives which are generated by the decision makers are more relevant to them, different behavior may be expected for $S$ generated and experimenter generated materials. This was the second independent manipulation.

In addition to the choices, $S$ s separately evaluated the attractiveness of the features of the alternatives and the importance of their dimensions. These values were used as external parameters of the four proposed models. Additionally, these ratings were used for predicting choices by the weighted additive rule.

\section{Method}

\section{Subjects}

Eight students who agreed to participate in 22 individual two hour sessions in one week intervals served as $S$ s. They were paid DM 5.- per hour and an additional DM 5.per hour only after completion of all sessions. The data of four other $S$ s who prematurely quit the experiment between the fourth and eight session are not reported here.

\section{Materials}

Four different choice domains with nine alternatives each were employed. The four domains were vacation areas, journal subscriptions, car rentals, and German universi-

[1] Originally, we had also considered a stochastic dimension selection model with $p_{i}$ directly proportional to $w_{i}$. This was not pursued further, because it introduced too much fluctuation in the predicted choices. 
ties. For each area, nine real alternatives were selected. These alternatives were then described by their features on eleven dimensions. A typical example of a named and a described choice pair is shown in table 1.

In addition, the $S$ s themselves generated alternatives, dimensions, and features for two of the choice domains.

\section{Design}

The alternatives of a domain could be presented in one of four different ways, obtained by crossing the two experimental variables, generation ( $S$, experimenter) and presentation mode (named, described). Each $S$ was run under all four of these conditions with one domain assigned to each condition. The assignment of domains to conditions was balanced over $S$ s. The nine alternatives of a domain form 36 choice pairs, yielding a total of 144 pairs of alternatives for the four domains. The $S \mathrm{~s}$ ' preferential choices from each pair were repeatedly elicited.

\section{Procedure}

All $S$ s were run individually. The experiment consisted of 22 sessions. In the first session, $S$ s generated nine alternatives for each of the two choice domains assigned to $S$-generated conditions. For one of these two domains they also generated 11 dimensions and the features of the alternatives of this domain on these dimensions.

For the remainder of the first session, unrelated materials were used for having the $S$ s practice the choice and evaluation tasks which were to follow. This exercise as well as the following 20 sessions were run under the control of an Apple II micro-computer and were completely $S$ paced.

Each of the 20 subsequent 1.5 to 2 hour sessions consisted of a choice and an evaluation part. The choice part consisted of the 144 paired comparisons of the nine

Table 1

Sample choice pair (journal subscriptions, experimenter-generated) in named and described presentation mode.

\begin{tabular}{lcc}
\hline Named presentation: & Capital & Spiegel \\
\hline Described presentation & & \\
\hline Frequency of publication & Monthly & Weekly \\
No. of articles on science & 4 & 1 \\
No. of advertisements & 120 & 180 \\
Price of magazine & $6 .-\mathrm{DM}$ & $4 .-\mathrm{DM}$ \\
No. of articles on politics & 70 & 25 \\
No. of articles on ecology & 1 & 5 \\
No. of articles on entertainment & 5 & 3 \\
No. of articles on cultural events & 3 & 5 \\
No. of pictures & 15 & 15 \\
No. of color pictures & 10 & 3 \\
No. of pages & 350 & 300 \\
\hline
\end{tabular}


alternatives from each of the four domains. The order of the pairs was mixed over domains and conditions and randomly determined for each session. For each comparison the following informations were simultaneously presented on a screen. At the top of the screen appeared the name of the domain. In the named conditions the two alternatives' names appeared on the screen, one beside the other. If the domain was assigned to a described condition the list of dimensions appeared, one below the other, on the left side of the screen. Their order was newly randomized for every new display. The alternatives' features on the dimensions were displayed in two columns to the right of the dimension names; alternatives' names were not given. The columns of the two alternatives of each pair were randomized over sessions.

The $S \mathrm{~s}$ indicated their choices by pressing one of two buttons. $S$ s were instructed to work fast but to take all the time they needed to make good decisions. The choices and choice latencies were recorded by the computer.

The evaluation part with 220 evaluation tasks followed. The $S$ s gave evaluations of the importance of dimensions and of the attractiveness of the alternatives' features for the two domains that were used in the described conditions. All these evaluations were done on seven point rating scales with the extreme poles being labelled "not important" and "very important" or "not attractive" and "very attractive". The items were randomly ordered within sessions. Each importance evaluation started with the text "How important is the following dimension for you in choosing among (domain)?" appearing on the screen. A similar text indicated domain and dimension when an attractiveness rating was required. Simultaneously the dimension or feature appeared on the screen. Below appeared a 7-point rating scale on which the $S$ s indicated their evaluation by turning a dial. The $S$ s' ratings were collected by the computer.

In the 22nd session, the $S$ s generated the dimensions and the alternatives' features on these dimensions for the named $S$-generated domain. Then evaluations for the two domains used under named conditions were elicited in this last session. This was done the same way as for described alternatives in the foregoing sessions but only once.

Data of one session were lost for four $S$ s (Nos. 3, 4, 5, 6) due to a discette failure. These $S$ s' results are based on only 19 replications.

\section{Results}

All analyses were done individually for each $S$ and domain. Table 2 shows a sample pattern of relative choice frequencies ordered by marginal frequencies. Typical features of the observed choice frequencies are the large numbers of zeros and ones and a band of probabilities different from zero and one around the diagonal. In order to test whether the relatively few non-zero/one frequencies were based on $S$ s' strategy changes during the earlier replications we compared their choice frequencies from the first half of replications with those from the second half. There was no significant increase of zeros and ones from first to second half.

\section{Fitting the choice models}

Each of the four models (ordinal and cardinal, VFL and SDS) was individually fitted to the observed choice frequencies for each $S$ and domain. For every $S$ and each 
domain the parameter $k$ was estimated by a grid search. In this estimation all integers from one to the highest accumulated difference that could occur were considered as possible parameters. For each model, the value of $k$ producing the minimal average of squared differences between observed and predicted choice probabilities was taken as the critical value.

Choice predictions with cardinal and ordinal VFL models were calculated from the $S \mathrm{~s}$ ' evaluations as was described in the introductory illustration. For domains used in described conditions, 20 sets of evaluations had been elicited. These were used to predict the choices repeatedly by using each set of values, in order to reflect the possibility that choice fluctuations could be accounted for by fluctuations in values.

Ordinal and cardinal SDS model predictions were derived from each $S$ 's averaged evaluations. These models were formalized as finite Markov chains. According to our assumption of a stochastic process for selecting dimensions, the value of the counter after each step of processing is a random variable. At every step there is a probability that the counter is at least as large as the positive critical value, $+k$, at least as small as $-k$, or absolutely smaller. In the first two cases one or the other alternative will be chosen. In the third case another dimension will be processed. Summing the probabilities that the counter is at least as large as $+k$ over all steps of the process yields the probability for choosing one alternative. The probability for choosing the other alternative is given by summing the probabilities of the counter being at least as small as $-k$. There may remain a probability that all dimensions are processed without reaching $+k$ or $-k$. For this case, choice was assumed with probability proportional to

Table 2

Observed choice frequencies (upper entries) and predicted choice probabilities (lower entries) for subject 3, vacation areas (described subject-generated alternatives). The predicted probabilities are from the cardinal SDS model with a critical value of 3 . The probabilities indicate preferences for column over row alternatives. Observed values are based on 19 observations and rounded.

\begin{tabular}{lllllllll}
\hline Alternative & 5 & 9 & 4 & 3 & 7 & 2 & 6 & 8 \\
\hline 9 & 0.80 & & & & & & & \\
4 & 0.58 & & & & & & & \\
3 & 0.95 & 0.65 & & & & & & \\
& 0.78 & 1 & & & & & & \\
7 & 1 & 0.75 & 0.55 & & & & & \\
& 0.80 & 0.73 & 0.56 & & & & & \\
2 & 1 & 1 & 0.95 & 0.90 & & & \\
6 & 0.93 & 1 & 0.85 & 0.83 & & & \\
& 1 & 1 & 1 & 0.85 & 0.95 & & & \\
8 & 1 & 1 & 1 & 0.82 & 1 & & & \\
& 1 & 1 & 1 & 1 & 1 & 1 & & \\
1 & 1 & 1 & 1 & 0.96 & 0.98 & 0.94 & & \\
& 1 & 1 & 1 & 1 & 1 & 1 & 0.90 & \\
& 0.98 & 1 & 0.96 & 1 & 1 & 0.80 & 0.25 & \\
& 1 & 1 & 1 & 1 & 1 & 1 & 1 & 1 \\
\hline
\end{tabular}


the counter's differences from $+k$ and $-k$. At any particular state in the process, the order in which the already processed dimensions were considered is irrelevant. Thus, by defining the set of possible states by the power set of all dimensions the Markov property is fulfilled for these states. A state becomes absorbing if a critical value is reached or exceeded in that state. For nonabsorbing states the transition probabilities to all other states that include one more dimension are positive and defined by the BTL-equation above. All other transition probabilities are zero.

\section{Model fit}

Table 3 displays the average standard deviations of the four models for the four experimental conditions. For comparison, the values for the weighted additive model using all dimensions are also reported. The additive model uses the assessed importance evaluations as weights for the attractiveness values rather than for determining the sequence of processing dimensions.

Altogether, our models fit the $S$ s choices better than the additive model. Further, table 3 shows no considerable differences in the pattern of model fits between $S$ - and experimenter-generated alternatives. But model fits are generally worse for named alternatives than for described ones. We will not consider named alternatives further.

For described alternatives, the cardinal SDS model fits the choices better than the ordinal one. This also holds on an individual basis for all eight $S$ s and for $S$ - as well as for experimenter-generated alternatives. For the VFL models there appears to be no difference between cardinal and ordinal models. Also, on an individual basis each is better in half the cases under both generation modes with individual differences being small. Therefore, we will compare the two cardinal models.

In order to give an impression of the individual variation, table 4 displays the individual model fits for cardinal models and described alternatives and the $S$ s' best fitting $k$ values. The cardinal SDS model is better than the cardinal VFL model in all but two cases. The probability of obtaining this superiority by chance is less than 0.002 by a binomial test. In seven cases, marked with an asterisk in table 4, the SDS model is also significantly better on an individual $S$ basis ( $F$-test on the arcsin transformed frequencies; Mosteller 1951; individual $p<0.05$ ). For further illustration, the predict-

\section{Table 3}

Average standard deviations of observed choice frequencies from the predicted ones for cardinal and ordinal VFL and SDS models and the weighted additive model (ADD) in the four experimental conditions.

\begin{tabular}{|c|c|c|c|c|c|c|c|}
\hline \multirow{3}{*}{$\begin{array}{l}\text { Alter- } \\
\text { natives }\end{array}$} & \multirow{3}{*}{$\begin{array}{l}\text { Comparison } \\
\text { modes }\end{array}$} & \multicolumn{6}{|c|}{ Alternatives generated by } \\
\hline & & \multicolumn{3}{|c|}{ Subject } & \multicolumn{3}{|c|}{ Experimenter } \\
\hline & & VFL & SDS & ADD & VFL & SDS & ADD \\
\hline \multirow[t]{2}{*}{ Described } & Cardinal & 0.28 & 0.20 & 0.35 & 0.27 & 0.20 & 0.31 \\
\hline & Ordinal & 0.29 & 0.27 & & 0.26 & 0.30 & \\
\hline \multirow[t]{2}{*}{ Named } & Cardinal & 0.37 & 0.32 & 0.47 & 0.40 & 0.32 & 0.49 \\
\hline & Ordinal & 0.39 & 0.31 & & 0.44 & 0.34 & \\
\hline
\end{tabular}


ions of the cardinal SDS model for $S 3, S$-generated alternatives, are shown in table 5 (lower entries).

\section{Choice latencies}

The models also predict the number of dimensions to be processed for choosing among each individual pair of alternatives. Therefore, the monotonicity of the function relating the observed choice latencies to the predicted number of dimensions was tested using the $\tau$-correlation. Only results for described alternatives and cardinal models will be reported. For named alternatives, there was no such relation.

For the VFL model this test is simple since this model predicts precisely how many dimensions will be processed for every pair. For each individual $S$ and domain the observed choice latencies were averaged over all choice pairs with identical numbers of predicted dimensions.

The SDS model does not predict a single number of dimensions but two probability distributions over number of dimensions for each pair, one for the choice of each alternative. Therefore, we calculated the following test quantities $\bar{t}_{m}, m=1, \ldots, 12$, where 12 refers to the case that the critical value is not reached after processing all 11 dimensions. Assume that a $S$ chooses $x$ from the pair $(x, y)$ in the $i$ th replication with latency $t_{x y i}$, and let $f_{x y m}$ be the model's probability that $x$ is chosen from pair $(x, y)$ after the processing of $m$ dimensions. Then

$\bar{t}_{m}=\left(\sum_{i=1,20} \sum_{(x, y)} t_{x y i} f_{x y m}+t_{y x i} f_{y x m}\right) /\left(20 \sum_{(x, y)} f_{x y m}+f_{y x m}\right)$

should be a monotone function of $m$. In simple words, this means that on the average choices should take more time, the more the probability is concentrated on higher numbers of dimensions.

Table 5 shows examples of the relation between predicted number of dimensions

Table 4

Individual subjects' critical values $(k)$ and model fits for cardinal models and described conditions.

\begin{tabular}{|c|c|c|c|c|c|c|c|c|}
\hline \multirow{4}{*}{$\begin{array}{l}\text { Sub- } \\
\text { ject }\end{array}$} & \multicolumn{8}{|c|}{ Alternatives generated by } \\
\hline & \multicolumn{4}{|c|}{ Subject } & \multicolumn{4}{|c|}{ Experimenter } \\
\hline & \multicolumn{2}{|l|}{$k$} & \multicolumn{2}{|c|}{ Model fit } & \multicolumn{2}{|l|}{$k$} & \multicolumn{2}{|c|}{ Model fit } \\
\hline & VFL & SDS & VFL & SDS & VFL & $\overline{\text { SDS }}$ & VFL & SDS \\
\hline 3 & 3 & 3 & 0.21 & $0.14^{*}$ & 5 & 6 & 0.26 & 0.17 \\
\hline 4 & 6 & 8 & 0.19 & $0.13^{*}$ & 3 & 8 & 0.31 & 0.26 \\
\hline 5 & 3 & 2 & 0.40 & 0.41 & 5 & 4 & 0.29 & $0.20^{*}$ \\
\hline 6 & 1 & 3 & 0.37 & 0.28 & 2 & 1 & 0.28 & $0.18^{*}$ \\
\hline 9 & 2 & 2 & 0.26 & 0.20 & 2 & 11 & 0.16 & 0.20 \\
\hline 11 & 3 & 1 & 0.18 & 0.15 & 4 & 5 & 0.25 & 0.20 \\
\hline 13 & 5 & 2 & 0.31 & $0.13^{*}$ & 5 & 3 & 0.29 & $0.16^{*}$ \\
\hline 14 & 2 & 1 & 0.31 & 0.22 & 1 & 3 & 0.35 & $0.22 *$ \\
\hline
\end{tabular}


Table 5

Predicted numbers of processed dimensions and observed average choice latencies for subject 3 (vacation areas) and 6 (universities), subject-generated domains. ( $m$ : numbers of dimensions processed by the models.)

(a) VFL model ( $f_{m}$ : number of pairs decided after processing $m$ dimensions;

$\bar{t}_{m}$ : average latency for these pairs).

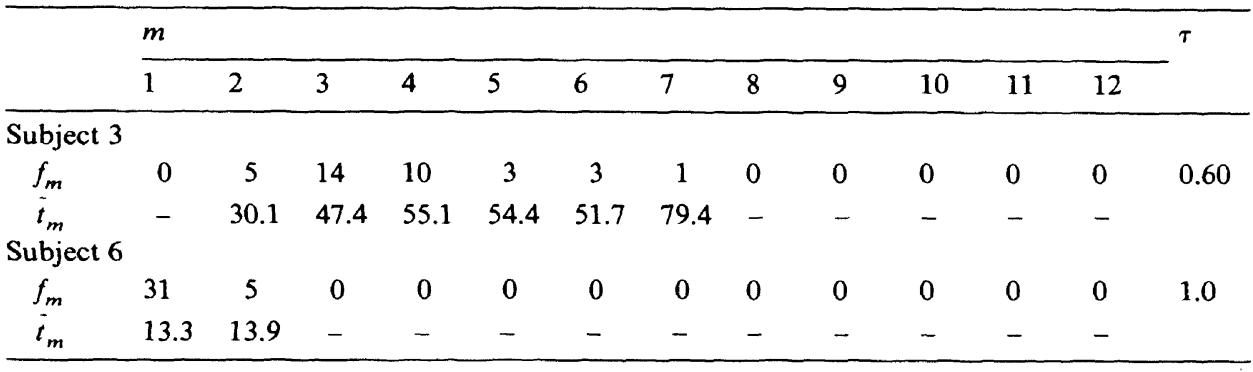

(b) SDS model ( $f_{m}$ : probability of processing $m$ dimensions;

$\bar{t}_{m}:$ weighted choice latency for $m$ dimensions).

\begin{tabular}{llllllllllllll}
\hline & $m$ & & & & & & & & & & & & \\
& 1 & 2 & 3 & 4 & 5 & 6 & 7 & 8 & 9 & 10 & 11 & 12 \\
\hline
\end{tabular}

\begin{tabular}{cccccccccccccc}
\hline Subject 3 & & & & & & & & & & & & & \\
$f_{m}$ & 0.03 & 0.19 & 0.18 & 0.14 & 0.11 & 0.09 & 0.06 & 0.05 & 0.04 & 0.03 & 0.01 & 0.07 & 0.97 \\
$\dot{t}_{m}$ & 41.2 & 43.3 & 43.0 & 46.0 & 48.9 & 51.1 & 52.3 & 55.4 & 55.9 & 58.1 & 63.8 & 66.3 & \\
Subject 6 & & & & & & & & & & & & & \\
$f_{m}$ & 0.37 & 0.22 & 0.12 & 0.07 & 0.05 & 0.03 & 0.02 & 0.02 & 0.01 & 0.01 & 0.01 & 0.07 & 0.55 \\
$i_{m}$ & 12.7 & 13.1 & 13.6 & 13.7 & 14.0 & 14.5 & 15.2 & 15.6 & 15.3 & 16.3 & 12.4 & 15.1 &
\end{tabular}

and observed latencies for two $S$ s and both models.

With three exceptions ( $S$-generated alternatives: $S$ 5, both models; $S$ 11, SDS model) all 32 (16 domains $\times 2$ models) $\tau$-correlations were positive. The median correlations for $S$ - and experimenter-generated conditions were 0.49 and 0.67 for the VFL model and 0.40 and 0.38 for the SDS model. Comparing the coefficients for the two models by a statistical test is not appropriate, however, for reasons of differences in the averaging formulas and because on the average the SDS model correlations are based on twice as many response time classes (11.6) as those of the VFL model (6.2).

\section{Discussion}

All together, the results for described alternatives support the assumption that binary choice is accomplished by sequential accumulation of dimensional value differences until enough evidence is accumulated. 
The models predict choices in various domains better than the additive model [2], and there is a positive relation between the subjects' choice latencies and the numbers of dimensions processed by the models.

Among our models, cardinal models accounting for value differences made better choice predictions than ordinal models. This result is in contrast to the fact that most choice heuristics described in the literature are ordinal. An explanation may be that we considered only binary choices. Payne (1976) observed that ordinal rules are mostly used to choose among more than two alternatives, whereas more elaborate comparisons are made between two alternatives [3].

From the cardinal models, the SDS model was found to be superior in predicting choices. This suggests that fluctuations in the process of selecting dimensions must be seriously considered as an alternative to fluctuations in values for explaining choice variability. An incidental observation about the choice latencies suggests, however, that our selection assumption may have introduced too much variation. The choice latencies of the SDS model of subject 6 in table 5 show that the violations of monotonicity occurred only for higher numbers of predicted dimensions which were assigned comparably low probabilities by the model. For the SDS model such low probability-high (7 to 11) numbers of dimensions choice latencies were the main source of violations of monotonicity among our subjects. This suggests that the subjects, contrarily to the SDS model, did not even occasionally use as many dimensions for making their choices. Further research on the process of selecting dimensions appears worthwhile.

For named alternatives, our models fitted less well. For named experimenter-generated alternatives this might have been explained by the subjects using other dimensions than we had assumed. Results were not better, however, in the subject-generated condition in which the subjects generated their own dimensions after making their choices. This suggests that the subjects did not use a dimensional strategy at all for choosing among named alternatives.

[2] Our models had an advantage over the additive rule in so far as one parameter was estimated from the choice data. No parameter was estimated from the choice latencies, however. Thus, the latency analysis may be considered as a cross validation favoring our models since the additive rule would not predict different latencies for individual pairs of alternatives.

[3] This explanation was suggested by Thomas Eppel. 


\section{Relation to other theories}

Our models incorporate a number of well known choice heuristics (that are discussed, for instance, by Svenson 1979, or Aschenbrenner 1981) as special cases, or they define probabilistic versions of these strategies. For instance, with a critical value of 1 the ordinal models become the lexicographic rule; with increasing critical values they approach the majority rule. The cardinal models with high critical values are equivalent to an additive model. Also, other modifications, not yet tested, would yield further established rules. For instance, one could assume that the comparison results are not accumulated over dimensions but that the counter is set back to zero after each dimension. With low critical values such cardinal models would become the lexicographic semiorder. With high critical values they might be considered as variants of the greatest attractivity difference rule with "greatest" being replaced by "sufficiently great".

Also, the general idea of describing choice processes by such models proposing a sequential information sampling process with a stopping criterion has already some tradition in psychology (e.g., Audley and Pike 1965). Elaborate versions of such models have been designed and applied for psychophysical judgment and discrimination tasks (e.g., Link 1975). Some support for such processes in multidimensional choice has been reported by Petrusic and Jamieson (1978). A sequential sampling model for one-dimensional (monetary outcome) risky choice was recently presented by Busemeyer (1982). Especially intriguing about such processes is the fact that they allow for a great reduction of information processing effort while yielding almost the same choice accuracy as can be achieved by processing all information about the alternatives.

The main difference of our approach is the use of externally assessed parameters (values and importance weights) for predicting choices. Also new is our solution for deriving the SDS model predictions from these parameters. This solution was needed as a consequence of the assumption that the same dimensions will not be processed more than once in any choice. Without this assumption, that is, when replacement of sampled information is assumed such models are formally much more tractable. 


\section{References}

Aschenbrenner, K.M., 1981. Efficient sets, decision heuristics, and single-peaked preferences. Journal of Mathematical Psychology 23, 227-256.

Audley, R.J. and A.R. Pike, 1965. Some alternative stochastic models of choice. British Journal of Mathematical and Statistical Psychology 18, 207-225.

Busemeyer, J.R., 1982. Choice behavior in a sequential decision making task. Organizational Behavior and Human Performance 29, 175-207.

Fishbein, M. and I. Ajzen, 1975. Belief, attitude, intention, and behavior. Reading, MA: AddisonWesley.

Huber, O., 1983. 'Dominance among some cognitive strategies for multidimensional decisions'. In: L. Sjoeberg, T. Tyszka and J.A. Wise (eds.), Human decision making. Lund: Doxa.

Link, S.W., 1975. The relative judgment theory of two choice response times. Journal of Mathematical Psychology 12, 114-135.

Luce, R.D., 1959. Individual choice behavior. New York: Wiley.

Mosteller, F., 1951. Remarks on the method of paired comparisons: III. Psychometrika 16, 208-218.

Payne, J.W., 1976. Task complexity and contingent processing in decision making: an information search and protocol analysis. Organizational Behavior and Human Performance 16, 366-387.

Petrusic, W.M. and D.G. Jamieson, 1978. Relation between probability of preferential choice and time to choose changes with practice. Journal of Experimental Psychology: Human Perception and Performance 4, 471-482.

Restle, F., 1961 Psychology of judgment and choice. New York: Wiley.

Svenson, O., 1979. Process descriptions of decision making. Organizational Behavior and Human Performance 23, 86-112.

Thurstone, L.L., 1927. A law of comparative judgment. Psychological Review 34, 273-286.

Tversky, A., 1972. Choice by elimination. Journal of Mathematical Psychology 9, 341-367. 